\title{
Sistema de formación docente como agente de cambio para el desarrollo humano: Caso Honduras
}

\author{
Dora Suyapa Díaz Quinteros \\ Doctora en Gestión del Desarrollo Humano en Ciencias Sociales \\ Docente investigadora en temáticas socio educativo \\ Universidad Nacional Autónoma de Honduras, Honduras \\ Email:ddiazq@yahoo.es
}

\section{Resumen}

Recepción: 5-10-2015 / Aceptación: 14-11-2015

En este artículo se analizan los resultados de la investigación realizada a docentes que laboran en los niveles de básica y media del sistema educativo hondureño en zona urbana y rural durante el año 2015. El propósito del estudio fue valorar el sistema de formación docente inicial y permanente en forma articulada con elementos científicos que contribuyan al conocimiento y la toma de decisiones. El trabajo hizo énfasis en: Sistema Formación Docente Inicial y Permanente, el Docente como agente de cambio, Teoría de Desarrollo Humano; todos estos aspectos empleados en categorías de análisis para sustentar la relación sustantiva entre el sistema de formación docente y la teoría desarrollo.

La verificación empírica fue obtenida por docentes en los departamentos de Honduras: Choluteca, Francisco Morazán, Lempira, Intibucá, Cortes, La Paz, Yoro. La muestra intencionada es de ciento siete docentes. En el trabajo de campo colaboraron estudiantes universitarios en colaboración con el Doctorado de Gestión del Desarrollo Humano en Ciencias Sociales de la Universidad Nacional Autónoma de Honduras y docentes del nivel desconcentrado.

Se encontró que el sistema de formación docente (SINAFOD) debe orientarse a conformar el plan nacional de formación de cambios, teniendo un hilo conductor a fin de identificar la identidad docente como facilitador en sus prácticas pedagógicas, que coadyuven al desarrollo humano en sus capacidades; todo esto articulado a la estratificación de formación para docentes nóveles, intermedios y experimentados; con la finalidad de un plan de seguimiento y evaluación a su desempeño, con base a áreas curriculares y sentidas del sistema y del contexto de la realidad educativa latente. Además, que las autoridades educativas expongan sobre la mesa de discusión y reflexión esta temática, a fin de tomar las decisiones más acertadas al respecto. En este sentido, el estudio utilizó como instrumento metodológico técnicas cuantitativas y cuantitativas como el cuestionario tipo encuesta en línea, entrevistas a investigadores en educación, las cuales contienen una serie de datos académicos. También se realizó una exhaustiva revisión de documentos teóricos y documentos públicos de la Secretaría de Educación, entre otros (SE).

Palabras clave: Sistema de Formación docente, docente como agente de cambio, Desarrollo humano.

\begin{abstract}
In this article the results of research conducted to teachers working in primary and secondary levels of the Honduran education system in urban and rural areas during 2015 are discussed The purpose of the study was to assess the system of initial teacher education and continuing in an articulated manner with scientific elements that contribute to knowledge and decision making. The work emphasized: Initial Teacher Education and Lifelong System, the Teacher as a change agent, Human Development Theory; all these aspects into categories of analysis used to support the substantive relationship between the system of teacher training and development theory.
\end{abstract}

The empirical verification was obtained by teachers in the departments of Honduras: Choluteca, Francisco Morazán , Lempira , Intibucá, Cortes , La Paz, Yoro. The purposive sample is one hundred and seven teachers. In the work field they helped university students in collaboration with the PhD in Human Development Management in Social Sciences of Universidad Nacional Autónoma de Honduras and teachers of the decentralized level.

We found that teacher training system (SINAFOD) should aim to shape the national training plan changes, taking a thread to identify teachers' identity as a facilitator in their teaching practices that contribute to human development in their abilities; this stratification articulated training for novice, intermediate and experienced teachers; in order to plan monitoring and evaluation of their performance, based on curricular and heartfelt system areas and the context of educational reality latent. In addition, the education authorities exposed on the table for discussion and reflection this subject, in order to make better decisions thereon. In this sense, the study used as a methodological tool quantitative and quantitative techniques such as online questionnaire survey, interviews with researchers in education, which contain a number of academic data. A comprehensive review of theoretical papers and public documents of the Ministry of Education, among others (SE) was also performed.

Key Words: System of Teacher Training, Teacher as an agen $t$ of change, Human Develpment 


\section{Introducción}

La educación es un aspecto puesto en la mesa de discusiones en la que los sectores sociales ligados al quehacer educativo pueden ver el futuro con optimismo hacia el desarrollo humano. En cada etapa de nuestra historia, la educación ha recobrado su centralidad en las agendas de trabajo de los distintos gobiernos a nivel mundial. La Educación y el Desarrollo son actualmente categorías en las cuales se discuten y planifican las prioridades de la humanidad, de cara a los cambios estructurales de los avances tecnológicos y científicos en un mundo cada día globalizado.

Esto se debe en buena medida al uso de la investigación para la «toma de decisiones» en el planteamiento de políticas educativas que contribuyan al desarrollo humano. Se podría mencionar que la toma de decisiones realizadas en las últimas décadas acerca del uso de investigaciones y sobre las contribuciones de la educación para el desarrollo, es establecerle a ésta un rol relevante en planes, programas de formación docente, proyectos sociales etc.

Un aspecto de interés en este estudio es la formación docente, con la finalidad de buscar el ámbito disciplinar, y el contexto social en que tiene lugar la enseñanza, apoyándose en estrategias formativas diversas y flexibles, para poder responder a las múltiples y diferentes necesidades individuales, disciplinares y contextuales con las que se enfrenta el docente. $\mathrm{Al}$ respecto Carlo Marcelo (2011) argumenta el papel del profesorado sigue siendo crucial, a pesar del amplio desarrollo de los recursos tecnológicos disponibles hoy en día, resulta fundamental plantearnos de nuevo de qué manera conseguimos que el profesorado se siga enamorando de su profesión y siga contribuyendo al desarrollo de sus escuelas y de los alumnos,

para fundamentar la idea de subsistemas articulados, un documento de la OCDE expone:

Las etapas de formación inicial, inserción y desarrollo profesional deberían de estar mucho más interrelacionadas para crear un aprendizaje coherente y un sistema de desarrollo para los profesores...Una perspectiva de aprendizaje a lo largo de la vida para los profesores implica para la mayoría de los países una atención más destacada a ofrecer apoyo a los profesores en sus primeros años de enseñanza, y en proporcionarles incentivos y recursos para su desarrollo profesional continuo. En general, sería más adecuado mejorar la inserción y el desarrollo profesional de los profesores a lo largo de su carrera en lugar de incrementar la duración de la formación inicial (OCDE, 2008a, 13).

Para cumplir estas tareas que cada día exige la sociedad en general se necesita un sistema de formación cualificado, no solo en el dominio científico y metodológico, sino que su identidad como docente esté apto a desarrollar un proceso de enseñanza en capacidades para la sociedad. Se sabe que la enseñanza es una actividad compleja y amplia, en la que inter- 
vienen multitud de categorías o variables para las que no existen recetas, pero esto no significa que no se pueda construir un sistema de formación desde las necesidades sentidas y prescriptivas por los docentes, pensando en el trabajo de forma desfragmentada, sin ninguna articulación de subsistema de formación docente y permanente.

Los sistemas de formación significan estimular al profesorado para que, como profesionales de la docencia, tiendan a centrar su labor en ejes: reflexión sobre la práctica; trabajo en equipo docente: comunidad de práctica y aprendizaje; y concebir y gestionar la enseñanza desde el aprendizaje. En una investigación en América Latina se concibe que uno de los aspectos a promover...

son programas de inserción a la docencia como una de las opciones de política más interesantes para asegurar un buen desempeño. La forma como se aborde el período de inserción tiene una importancia trascendental en el proceso de convertirse en un profesor. Además este tema podría devenir una puerta de entrada para articular formación inicial y desarrollo profesional docente. Se trata de una temática nueva, muy pocos son los países en América latina que tienen definida una política en la materia. Esto tiene la ventaja de hacer que las autoridades, los hacedores de políticas, los investigadores piensen el tema articuladamente con otros asuntos como el referido a la carrera y el desarrollo profesional, a la evaluación y a las remuneraciones (Denise Vaillant, 2007).
En este sentido, las iniciativas de innovación integrados en enfoques educativos a nivel mundial apuestan a inversiones en los sistemas educativos, siendo una de las tareas específicas de estos sistemas la formación docente; aspecto fundamental para definir políticas públicas a un modelo de sociedad que se acerque al desarrollo humano en capacidades, y lograr el bienestar común que hoy en día tanto se menciona. Pero todas las diversas iniciativas se pueden reducir a una sociedad global que expande su economía a través de la innovación, el saber y la sustitución del empleo de baja capacitación por procesos automáticos expandidos. También, todas las conceptualizaciones se orientan a definir el conocimiento como el factor clave y motor central de la nueva sociedad. (Rama, C., 2007, p.7)

La innovación en el conocimiento a este cambio es también partir del supuesto básico de que los docentes ni son culpables de los problemas de la educación actual (sin que por eso sean inocentes), ni son o pueden ser los actores singulares del mejoramiento de la educación, pues son muchos los factores que contribuyen a producir sus resultados. Andy Hargreaves (2007) contempla la necesidad de nuevas destrezas y cualidades de la sociedad post-industrial y la de modernas estructuras escolares que las generen. Es evidente que un objetivo importante de la educación en una sociedad post-industrial es educar a los jóvenes en destrezas y cualidades como la adaptabilidad, la flexibilidad y la capacidad para 
trabajar con otros. Lo mismo ocurre respecto a la familiarización con las nuevas tecnologías que caracterizarán cada vez más muchos ambientes laborales. «Todo esto pone manifiesto la necesidad de ambiente escolares que puedan generar el aprendizaje autónomo, individualizado y significativamente cooperativo, esencial para el puesto de trabajo posindustrial». ${ }^{1}$

En resumen, el asunto de la formación docente es un tema que conlleva a pensar sobre el rol fundamental de cambio que posee el educador, en el desarrollo humano de una sociedad. Quizá esto no sea una respuesta a toda esa complejidad, pero la finalidad es tomar decisiones con base a necesidades de capacitaciones, articular los sistemas, y un esfuerzo conjunto de instituciones formadoras que conlleven un hilo conductor hacia programas de capacitación para docentes noveles, docentes con experiencias intermedias y docentes con un recorrido de experimentados. En Honduras esta necesidad se hace más latente y más compleja, por contar con una representatividad muy grande en unidocencia.

\section{Al respecto Díaz Quintero (2010) afirma:}

nos enteramos que la unidocencia en Honduras es una realidad educativa que está latente y que muy pocas veces las autoridades educativas han expuesto sobre la mesa de discusión y reflexión, a fin de tomar las decisiones más acertadas al respecto. Esta realidad de escuelas unidocentes refleja un $38 \%$ y un $26 \%$ de escuelas bidocentes, estableciendo un alarmante $64 \%$ de la totalidad de escuelas del país. En este sentido, el estudio utilizó como instrumento metodológico técnicas cualitativas como las de grupos colaborativos, fichas de observación y entrevistas, las cuales contienen una serie de datos académicos, así como la caracterización del profesor unidocente que labora en este estrato; además, se realizó también una exhaustiva revisión de documentos en los archivos de la Secretaría de Educación (SE). ${ }^{2}$

Aunado a la problemática particular de Honduras, vale la pena preguntarse ¿por qué no se puede implementar lo que se ha construido? ¿Por qué se ven las cosas en forma desfragmentada, desarticulada y solo durante periodos de gobierno, no como una política de Estado?

La realidad de los indicadores educativos condena esta realidad cada día, cuando se argumenta y acepta esta realidad, cayéndose en un círculo vicioso en donde se ejecutan medidas paliativas, y no se ataca la raíz. Cada día se argumenta que la formación docente debe ser orientada en forma integral - reflexión válida -, pero ¿cuándo el cambio? La ilustración de $\mathrm{x}$ o y teórico..., las reglas del mundo están cam-

1. Hargreave, A. S. (2003). Profesorado, cultura y postmodernidad, Cambian los tiempos, cambia el profesorado; Ediciones Morata, SL. 76,77.

2. Diaz. (2010). La unidocencia: desafíos y retos con la aplicación del diseño curricular nacional básico (DCNB) en la formación permanente en el marco del sistema nacional de formación docente (SINAFOD). Revista Centro Americana de Educación; Patria Grande, CECC- SICA. 
biando. Es hora de que las reglas de la enseñanza y del trabajo de los docentes varíen con ellas. Las reglas del mundo están cambiando, en palabras de Andy Hargreaves (2007), «El autor en mención, afirma que la enseñanza y el trabajo de los docentes está siendo afectada en la medida que en que se modifiquen las condiciones y los valores sociales...».

Partiendo de estos argumentos, formación docente significa poseer la convicción en posibilitar su ejecución, tal como fue construido, del cual el referente investigativo expuesto en este artículo, sustenta un plan nacional de formación en Honduras; que por cierto, ya hace años han comenzado en algunos técnicos, a través de las oportunidades de trabajo en la Secretaria de Educación, elaborando el SINAFOD: Sistema Nacional de Formación Docente SINAFOD; concibiéndose como el conjunto de instituciones, organismos y recursos que, en forma integrada y coordinada, actúan en la formación inicial y permanente de los docentes. Esto atiende a las políticas nacionales, sus objetivos, las estrategias de desarrollo y los planes estratégicos del Sistema Educativo de Honduras. ${ }^{3}$

En este sentido, no solo son acertados sino extrapolables a al actual contexto en sus dimensiones fundamentales, la centralización de los cambios en el quehacer educativo relacionado a políticas de gobierno por periodos de cuatro años. Para el caso de Honduras se han generado cambios que causan rebeldía y afectan a la enseñanza, a causa de no ejecutar un sistema de formación sistémico, entre formación inicial (Universidades) y formación permanente (Institutos de formación), donde se articulen los subsistemas y se regule el proceso de formación en cada uno de estos. Para ello se debe hacer una interjección de un conjunto de acciones llevadas a un proceso integrado, apostándole a ser competentes para la enseñanza, la actitud, la aptitud, y a cómo el docente puede ser agente de desarrollo a través de la enseñanza.

Andy Hargreaves aglutina su análisis respecto a los retos que la postmodernidad plantea al profesorado: el trabajo, el tiempo y las culturas de la enseñanza. El trabajo de los docentes está sujeto a demandas que, a menudo, son contradictorias entre sí; que cambian y que suelen configurar imagen de incertidumbre y un incremento de los riesgos en la toma de decisiones. ¿Cuál es el sentido de la escuela y qué papel ha de jugar respecto a la sociedad? ¿Cuál el sentido de los diferentes niveles educativos? ¿Qué conocimiento es importante? ¿Cómo incorporar los problemas sociales relevantes? ¿Cómo proporcionar una formación que integre la vinculación que sea accesible a todos los actores de la educación? ¿Se seguirá siendo "profesores" o se convertirán en "entretenedores" de adolescentes? ¿En qué parcelas de decisión puede intervenir el alumnado, los padres y madres?

3. Documento extraído del Sistema Nacional de Formación Docente.(2009 )INICE, p 4, Honduras 
A los cambios cualitativos - los que tienen que ver con la función social de la escuela, las nuevas directrices curriculares, las modificaciones en el propio papel de los docentes - se añaden también nuevas tareas a realizar, colectiva e individualmente. Esto genera lo que se ha venido denominando "intensificación": un incremento de las demandas que implica reducción del tiempo que los docentes dedican a funciones "educativas" y una saturación de tareas administrativas y burocratizadas. En definitiva, fuertes modificaciones en la estructura del puesto de trabajo que también el docente afronta y afecta a su ejercicio, al cambio de su disciplina; aunado a otros factores que definitivamente no pueden obviarse en el salón de clase como el reflejo de la desigualdad y exclusión social, que se describe cada día en el aula, por tener una idea de este tema adyacente en Honduras.

$\mathrm{Al}$ respecto, un estudio presentado por los hondureños Alas y Mocada (s.f.),

se aborda el tema de la calidad y equidad en la educación básica y media de la educación nacional. Se constata que los actuales esfuerzos para mejorar la equidad de la educación en América Latina en general y en Honduras en particular, se enfocan en la búsqueda de intervenciones que, gestionando los escasos recursos disponibles, disminuyan las brechas en el acceso y el desempeño escolar asociados a las diferencias por estratos socioeco- nómicos, grupos étnicos o por región de residencia de los estudiantes. El análisis concluye que, en el marco de profundas desigualdades sociales, el sistema educativo hondureño ha avanzado poco en la búsqueda de la equidad en educación, pese al desarrollo de varias y costosas intervenciones durante las últimas dos décadas. Finalmente se plantea la urgencia de reorientar las políticas educativas de equidad. ${ }^{4}$

Carol H. Weiss (2009) argumenta, ${ }^{5}$

La urgencia de esta cuestión es fácil de ver. Para los investigadores y las organizaciones que los financian, el objetivo global de la investigación para el desarrollo es mejorar la vida de las personas en los países en desarrollo. Muy a menudo, las políticas públicas son un instrumento indispensable para transformar el conocimiento nuevo en una vida y un futuro mejores;

y la urgencia es igualmente apremiante para la comunidad política. Al fin y al cabo, el acceso sistemático al asesoramiento sobre investigación - basada en pruebas - puede mejorar espectacularmente las posibilidades de decidir y llevar a cabo medidas políticas que obtengan los resultados buscados y atraigan apoyo público duradero. Los investigadores y los políticos no siempre utilizan el mismo lenguaje. Pero pueden encontrar una causa común en la búsqueda de una política relativa al desarrollo que sea justa y sostenible. El acuerdo sobre una causa común así no es

4. Alas, M. y Moncada, G. (s.f.). Calidad y equidad en la educación nacional; Estado de la educación nacional, U.P.N.F.M. p 79. 5. Weiis C. H. (2009). Del conocimiento a la política .Máximo aprovechamiento de la investigación para el Desarrollo. Editorial Barcelona. 
fácil ni inevitable, desde luego. Para los malos gobiernos, una gobernación justa y sostenible no es una prioridad; $y$ los buenos gobiernos luchan cada día para hacer cuadrar objetivos políticos diversos y aparentemente contradictorios. A lo sumo, la investigación sólo contará como una de entre muchas influencias en el proceso político.

Por su parte, no todos los investigadores se comprometen con la pertinencia política; algunos creen, muy honradamente, que la investigación consigue más cuando no está sometida a los objetivos políticos y puede seguir libremente sus propias indicaciones. Para los fines actuales, no obstante, se hace el supuesto, entre los políticos, al menos una disposición latente a escuchar algún consejo potencialmente provechoso $\mathrm{y}$, entre los investigadores, una disposición a darlo.

Según de Souza (2005), mirando más allá del "desarrollo", la red es una iniciativa que conforma dichas posibilidades, semejante al programa latinoamericano de investigación sobre Modernidad / Colonialidad, cuyo proyecto epistémico ético y político supera el racismo del colonialismo y universalismo de la modernidad para abrir otras ventanas - éticas políticas -epistemológicas- para innovación social, institucional, política y cultural. ${ }^{6}$

En síntesis, los cambios que afrontan tienen que estar ligados a esta problemática real, debido a que es de mucha complejidad y amplitud. La formación docente es un tema de valor agregado relacionado con la docencia, centrado en temáticas como: diseño curricular, psicología del aprendizaje, metodologías activas y colaborativas, evaluación, etc.); facilitando enormemente el trabajo a los equipos docentes. Quien sabe bien a dónde va, entiende lo que encuentra y se siente capaz no solamente de buscar soluciones adecuadas para los problemas que surgen, sino también de ser proactivo.

En la mayor parte de los casos, los docentes en la región - sin un sistema regulado - accedieron a sus cargos sin la formación necesaria. Sin embargo, al ser conscientes de esa carencia, han buscado los medios para completar o mejorar su formación, haciéndola extensiva al resto del profesorado. En los centros o servicios responsables de la formación del profesorado es frecuente la demanda de formación para un centro o para una titulación concreta, en temas «sentidos» como especialmente necesarios, al poner en práctica las guías docentes; destacando las metodologías activas y colaborativas, el trabajo en equipo y la evaluación (en este último caso la demanda es muy alta).

A ese respecto, es muy generalizada la preocupación de los educadores o quienes dirigen al constatar que, habitualmente, sólo tienen capacidad para abordar y solucionar de forma satisfactoria retos de un nivel no muy complejo; con frecuencia fruto de su eficaz gestión personal y de la colaboración del profesorado. Cuando los problemas afectan a nivel

6. De Souza Silva, J. (2005). La innovación de la innovación institucional: De lo universal, mecánico y neutral a lo contextual, interactivo y ético desde una perspectiva latinoamericana. 
institucional, departamental, nacional - sin mencionar estadísticas gubernamentales - las dificultades aumentan y no suele encontrarse una respuesta satisfactoria, generándose cierta confusión.

Al ser la cara de un país, la educación y los niveles de avance están desarticulados. Es preciso reflexionar ¿cómo se están formando los docentes? ¿Quiénes los forman? Es así como comienzan las acusaciones y se plantea un abanico de «reformas» o cambios, sin precisar una transformación sustancial y/o estructural al sistema de formación inicial y permanente; capacitaciones no fragmentadas, a docentes nóveles, intermedios y experimentados, orientado a lo prescrito en el marco de la nueva ley general de educación en Honduras.

Sin duda es un buen inicio para el desarrollo de políticas favorecedoras para gestar un sistema de formación docente estratificado, ${ }^{7}$ articulado con instituciones formadoras. Asimismo, orientado a destacar la importancia del papel que desarrollarían los programas de formación relacionados con la docencia, derivados del sistema. En todos ellos se fundamenta ampliamente su relevancia en el marco de una sociedad que demanda un verdadero cambio de modelo educativo en la educación plantea un tipo de formación menos vinculada a un proceso de selección fragmentado $\mathrm{y}$ más centrada a capacidades, competencias, actitudes, destrezas, etc. Del cual se geste un sistema de formación docente integral.
Finalmente, el trabajo de los docentes está sujeto a demandas que, a menudo, son contradictorias entre sí; que cambian y que suelen configurar imagen de incertidumbre y un incremento de los riesgos en la toma de decisiones. ¿Cuál es el sentido de la escuela, y qué papel ha de jugar respecto a la sociedad? ¿Cuál el sentido de los diferentes niveles educativos? ¿Qué conocimiento es importante? ¿Cómo incorporar los problemas sociales relevantes? ¿Cómo proporcionar una formación que integre la vinculación accesible a todos los actores educativos y a la profundidad? ¿Se sigue siendo «profesor» o se ha convertido a convertir en «entretenedores» de adolescentes? ¿En qué parcelas de decisión puede intervenir el alumnado, los padres y madres?

El tiempo es una dimensión. Se estructura en este enfoque un asunto crucial; el tiempo debe tener una administración con recepción racional, es decir mecanismos profesionales e institucionales de responsabilidad sobre el uso del tiempo, en unas condiciones de flexibilidad que permitan aunar la autonomía y responsabilidad, las necesidades colectivas de un centro y cada uno de sus miembros.

En la teoría de Carol H. Weiss, ${ }^{8}$ y basado en las primicias para su construcción, un referente teórico fundamental en el campo del desarrollo humano requiere de explicaciones y aproximaciones interdisciplinares. Los estudios sobre desarrollo están en plena revisión debido a los desafíos que le plantea el proceso 
de globalización (Hettne, 2002). Asimismo, los problemas específicos del desarrollo humano (Schuurman, 2002); actualmente, los desafíos del tercer mundo son otros. Lo que sí parece vigente es la necesidad de profundizar en la investigación sobre el desarrollo porque - a pesar de que hayan transcurrido más de cincuenta años desde que se empezará a teorizar sobre el mismo, y se definieran los primeros planes de desarrollo - los resultados a escala global son muy bajos y se sigue estando en grandes procesos de exclusión social.

El informe de Desarrollo Humano (2013) ${ }^{9}$ afirma que si se analiza de cerca la trayectoria seguida por exitosos países en desarrollo, se conocerán nuevas opciones de políticas posibles para todos los países regiones, y se aprenderá sobre valores y visiones del mundo que podrían propiciar acciones futuras de cooperación para el desarrollo; y respuestas constructivas ante los desafíos mundiales más graves. El objetivo, como siempre, es acelerar en la medida de lo posible el progreso a gran escala, el cual eleva el estándar y ofrece más oportunidades a las personas de todos los países y comunidades, en todas las dimensiones clave del desarrollo humano, desde salud, educación y subsistencia, hasta la libertad personal para controlar y mejorar la propia vida.

Este documento pretende indagar sobre cómo se están dando cambios en la educación, en las formas de enseñanza, las opcio-

9. Informe de Desarrollo Humano (2013) PNUD, p 11. nes y retos que se plantean en las prácticas educativas a medida que se avanza en la era postmoderna; el tipo de sociedad en la que se vive y las transformaciones características de la sociedad, que produce y producirá en el ser humano, haciendo uso de la investigación educativa. Es fundamental conocer si los cambios hechos y por hacer han influido, o si la investigación es o debe ser un núcleo para construir puentes en la educación, y sus cambios para el desarrollo humano en el quehacer cotidiano y los profundos cambios sociales complejos e, incluso globales, y también a los propios actores de la educación.

Asimismo, ubicar la mirada en el uso de la investigación educativa en el desarrollo humano es pensar y aplicar una interacción desde la perspectiva holística e interdisciplinar, profundizar el problema; es no pensar en hacer un documento de escritorio y no compartir la idea. ¿Para qué investigar?, sería muy difícil tomar decisiones acertadas. Otro punto del discurso, se concentra en revalorizar la investigación.

Uno de los propósitos de este estudio es ver el círculo virtuoso de la investigación en el vínculo investigativo en la toma de decisiones, desde niveles operativos hasta altos niveles, serían opciones para incidir en resolución de problemas en nuestro contexto. Partiendo desde la raíz, y con la construcción de iniciativas técnicas como el SINAFOD, el cual está fundamentado en los hallazgos que a continuación se presentan en vías de integrar un plan nacional de formación docente integral. 


\section{Metodología}

Investigación con enfoque cuantitativo, utilizando el cuestionario. Este instrumento capturó datos consistentes con planteamientos de preguntas básicas, directamente de los sujetos estudiados, a partir de la formulación de una serie de preguntas en línea; conteniendo ítems en escalas Likert de intensidad, siendo esta intensidad valorativa sumatoria de opinión. Se realizaron cruces de variables, preestableciendo un plan de análisis y estableciendo relaciones entre las mismas.

También se llevó a cabo un análisis documental, con enfoque cualitativo (fundamentalmente inductivo), de documentos oficiales legislativos y curriculares; y estudios realizados con la finalidad de ubicar la identidad del docente en el rol, como agente de cambio, desde un sistema de formación docente.
Por los niveles de Educación Básica y Media, ciclos y la diversidad de modalidades existentes en el sistema educativo que rectora la Secretaria de Educación, se estableció que la muestra fuera de tipo estratificada, por áreas y conglomerados. Los municipios y los centros educativos participantes correspondieron a un conglomerados de 50 docentes en línea y 57 aplicados, directamente, a centros educativos en los departamentos de: Choluteca, Francisco Morazán, Lempira, Intibucá, Cortes, La Paz, Yoro; con y sin formación universitaria ubicados en zonas urbanas y rurales (áreas).

Por su naturaleza, este muestreo fue teórico, ${ }^{10}$ orientado a un enfoque cualitativo donde se buscó acercarse a los sentimientos, pensamientos y acciones de los participantes en cuanto al fenómeno investigativo. A continuación se muestra un resumen sobre la composición de la muestra.

Tabla 1. Composición de la muestra

\begin{tabular}{|l|l|c|}
\hline Nivel & \multicolumn{1}{|c|}{ Estratos de los informantes } & Miembros por estrato \\
\hline \multirow{4}{*}{ Descripción } & $\begin{array}{l}\text { Docentes nóveles, Intermedios y experi- } \\
\text { mentados }\end{array}$ & \\
\hline & Básica: Subtotal & $\mathbf{6 7}$ \\
\hline \multirow{4}{*}{$\begin{array}{c}\text { Docentes noveles, inter- } \\
\text { medios y experimentados }\end{array}$} & Directores de Escuelas uni y bi docentes & \\
\cline { 2 - 3 } & Directores de centros educativos de I y II ciclo & \\
\cline { 2 - 3 } & Directores de Centros de Educación & \\
\cline { 2 - 3 } & Directores de centros educativos & \\
\cline { 2 - 3 } & Docentes del I ciclo & \\
\cline { 2 - 3 } & Docentes del II ciclo & \\
\hline & Dedia: Subtotal & $\mathbf{4 0}$ \\
\hline
\end{tabular}

10. El muestreo teórico es un método de investigación frecuentemente asociado con las ciencias sociales, como por ejemplo la psicología. Fue desarrollado por los sociólogos Barney Glaser (n.1930) y Anselm Strauss (1916-1996). 


\begin{tabular}{|c|c|c|}
\hline Nivel & Estratos de los informantes & Miembros por estrato \\
\hline \multirow{5}{*}{$\begin{array}{l}\text { Docentes noveles, inter- } \\
\text { medios y experimentados }\end{array}$} & Docentes de Ciclo Común & \\
\hline & Docentes de Ciclo Básico Técnico & \\
\hline & $\begin{array}{l}\text { Docentes de diversificado (bachillerato } \\
\text { en CCLL) }\end{array}$ & \\
\hline & $\begin{array}{c}\text { Docentes de diversificado (Bachillerato } \\
\text { técnico) }\end{array}$ & \\
\hline & Total & 107 \\
\hline
\end{tabular}

Nota: Docentes estatificados por años de servicio: Nóveles, itermedios y experimentados.

Tabla 2. Distribución de muestra por años de experiencia en docencia

\begin{tabular}{|c|c|}
\hline Años de servicio & Porcentaje \\
\hline Hasta 10 años & $40 \%$ \\
\hline De 11 a 20 años & $\mathbf{3 3} \%$ \\
\hline De 21 a 30 años & $16 \%$ \\
\hline De 31 a 40 años & $11 \%$ \\
\hline Total & $\mathbf{1 0 0} \%$ \\
\hline
\end{tabular}

Tabla 3. Distribución de muestra de zonas de trabajo

\begin{tabular}{|c|c|}
\hline Zona de trabajo & Porcentaje \\
\hline Urbano & $66 \%$ \\
\hline Rural & $\mathbf{1 6} \%$ \\
\hline Urbano marginal & $18 \%$ \\
\hline Total & $\mathbf{1 0 0} \%$ \\
\hline
\end{tabular}

En las tablas 2 y 3 se refleja la cantidad de do- Resaltan los docentes que tienen más de 20 centes que formaron parte de la muestra, dis- años de servicio, así como los que trabajan en tribuida por años de servicio y zona de trabajo. la zona urbana. 


\section{Resultados}

\subsection{Entorno laboral del docente}

Tabla 4. Autonomía para toma de decisiones

\begin{tabular}{|c|c|c|}
\hline \multirow{2}{*}{ Años de servicio } & \multicolumn{2}{|c|}{ Tengo aotonomía para tomar decisiones } \\
\cline { 2 - 3 } & De acuerdo & En desacuerdo \\
\hline Hasta 10 años & $53 \%$ & $15 \%$ \\
\hline De 11 a 20 años & $33 \%$ & $38 \%$ \\
\hline De 21 a 30 años & $9 \%$ & $38 \%$ \\
\hline De 31 a 40 años & $4 \%$ & $8 \%$ \\
\hline
\end{tabular}

La tabla refleja cómo, en la medida que los años de servicio aumentan, la autonomía en la toma de decisiones disminuye. Asimismo, el $53 \%$ de docentes se encuentran de acuerdo con este postulado, mientras que el 15\% manifestó estar en desacuerdo.

Tabla 5. Resolución de problemas

\begin{tabular}{|c|c|c|}
\hline \multirow{2}{*}{ Años de servicio } & \multicolumn{2}{|c|}{$\begin{array}{c}\text { Existe preocupación por la resolución de problemas en el } \\
\text { quehacer educativo }\end{array}$} \\
\cline { 2 - 3 } & De acuerdo & En desacuerdo \\
\hline Hasta 10 años & $47 \%$ & $30 \%$ \\
\hline De 11 a 20 años & $34 \%$ & $40 \%$ \\
\hline De 21 a 30 años & $14 \%$ & $23 \%$ \\
\hline De 31 a 40 años & $4 \%$ & $7 \%$ \\
\hline
\end{tabular}

Distintos procesos sociales imponen exigencias a la formación docente. Como se afirma en los planes nacionales de otros países, la formación docente debe tomar en cuenta los procesos en el entorno local de la escuela porque la educación requiere cada vez más cooperación entre los actores que están dentro y fuera de los establecimientos educativos.
En convenios internacionales se argumenta que, la educación básica y media enfatiza la igualdad y la prevención de la exclusión. Por eso, se considera fundamental la participación de toda la comunidad educativa (docentes, alumnos, padres etc.) en la toma de decisiones, y es cada vez más importante tomar en cuenta las diferencias personales y culturales de los alumnos. 


\subsection{Condiciones de desarrollo profesional}

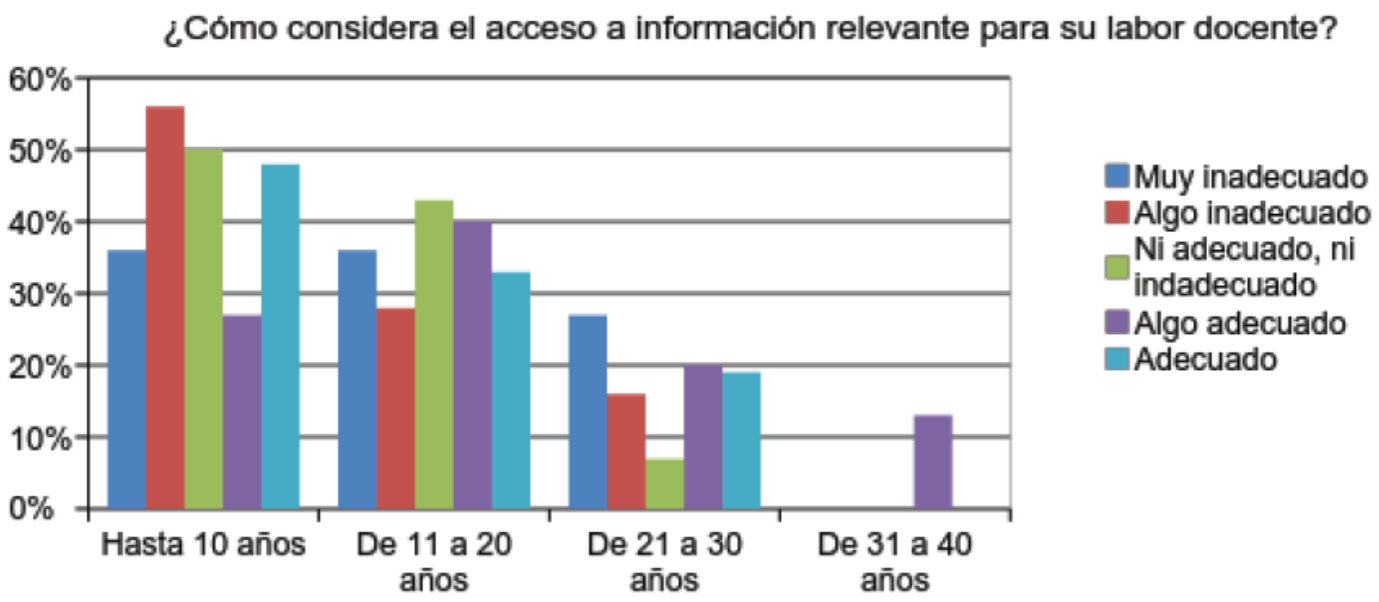

Figura 1. Información relevante para la labor docente.

De los docentes encuestados, que pertenecen al rango de 10 años de servicios, solo el 56\% considera algo inadecuado el acceso a información relevante, respecto a su labor docente. Un $43 \%$ de los docentes con 11 a 20 años de servicio no lo consideran ni adecuado, ni inade- cuado; mientras que solo el $27 \%$ de los docentes en el rango de 21 a 30 años lo considera muy adecuado. Llama la atención que en la última categoría (docentes de 31 a 40 años de servicio), el 13\% manifestó que es algo adecuado.

¿Cómo considera el sistema de promoción de grados subsiguientes en sus

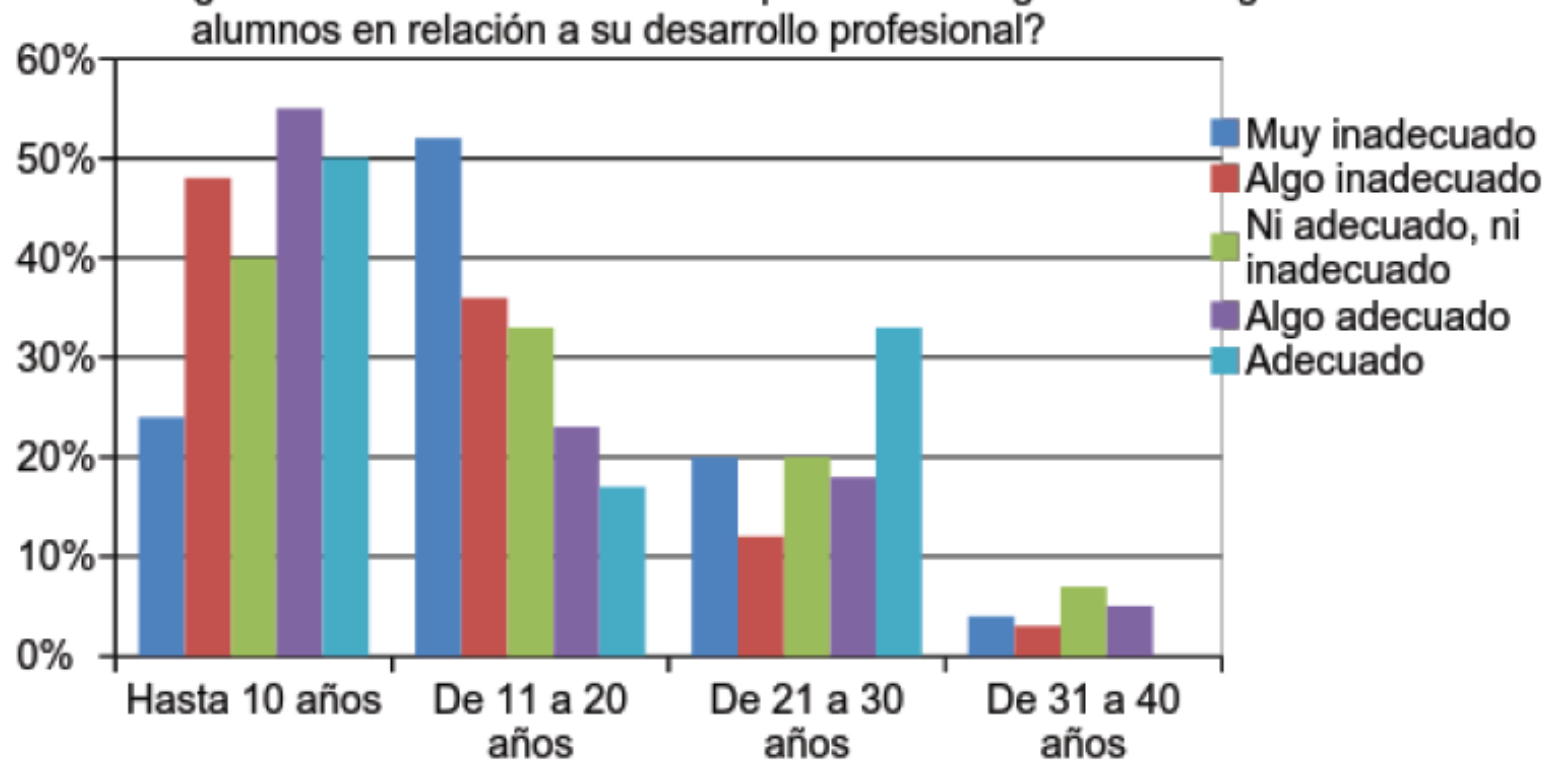

Figura 2. Respuestas de los docentes ante cuestionamientos sobre desarrollo profesional. 
Otros países cuentan con orientaciones de política que establecen referencias a la integración de Tecnologías de la información y comunicación social (TIC) en la formación inicial y permanente de docentes. Honduras ha tenido muy poca incidencia con programas como
«Educatrachos» para aprendizajes a escolares y alfabetización en TIC a docentes, entre otros. Pero no solo como una decidida política de integración de las TIC a la educación, a través de iniciativas y acciones de carácter nacional.

\subsection{Satisfacción del docente}

Tabla 6. Procedimientos de contratación

\begin{tabular}{|c|c|c|c|c|}
\hline \multirow{2}{*}{ Años de servicio } & \multicolumn{4}{|c|}{ Cómo considera los procedimientos de contratación para la inserción } \\
& \multicolumn{4}{|c|}{ a la docencia } \\
\cline { 2 - 5 } & Muy satisfecho & Satisfecho & Muy insatisfecho & Insatisfactorio \\
\hline Hasta 10 años & $50 \%$ & $52 \%$ & $44 \%$ & $36 \%$ \\
\hline De 11 a 20 años & $50 \%$ & $20 \%$ & $47 \%$ & $33 \%$ \\
\hline De 21 a 30 años & $0 \%$ & $20 \%$ & $6 \%$ & $28 \%$ \\
\hline De 31 a 40 años & $0 \%$ & $8 \%$ & $3 \%$ & $3 \%$ \\
\hline
\end{tabular}

Los porcentajes de satisfacción e insatisfacción para los docentes que tienen 10 años de servicio son constantes y no guardan mayor di- ferencia entre sí; pero a mayor tiempo de servicio menor es la satisfacción de contratación.

Tabla 7. La formación recibida para la práctica

\begin{tabular}{|c|c|c|c|c|}
\hline \multirow{2}{*}{ Años de servicio } & \multicolumn{4}{|c|}{$\begin{array}{c}\text { Cómo considera la formación inicial recibida para el desarrollo de } \\
\text { enseñanza en la práctica pedagógica }\end{array}$} \\
\cline { 2 - 5 } & Muy satisfecho & Satisfecho & Muy insatisfecho & Insatisfactorio \\
\hline Hasta 10 años & $35 \%$ & $47 \%$ & $27 \%$ & $50 \%$ \\
\hline De 11 a 20 años & $47 \%$ & $25 \%$ & $47 \%$ & $50 \%$ \\
\hline De 21 a 30 años & $18 \%$ & $22 \%$ & $20 \%$ & $0 \%$ \\
\hline De 31 a 40 años & $0 \%$ & $5 \%$ & $7 \%$ & $0 \%$ \\
\hline
\end{tabular}

Es notable como el paso del tiempo ha influido en el desarrollo de nuevas prácticas para el desarrollo de la enseñanza; lo anterior se deduce del nivel mínimo de satisfacción que manifiestan aquellos docentes con mayor tiempo de servicio. 
¿Considera que la práctica enseña la identidad de ser docente?

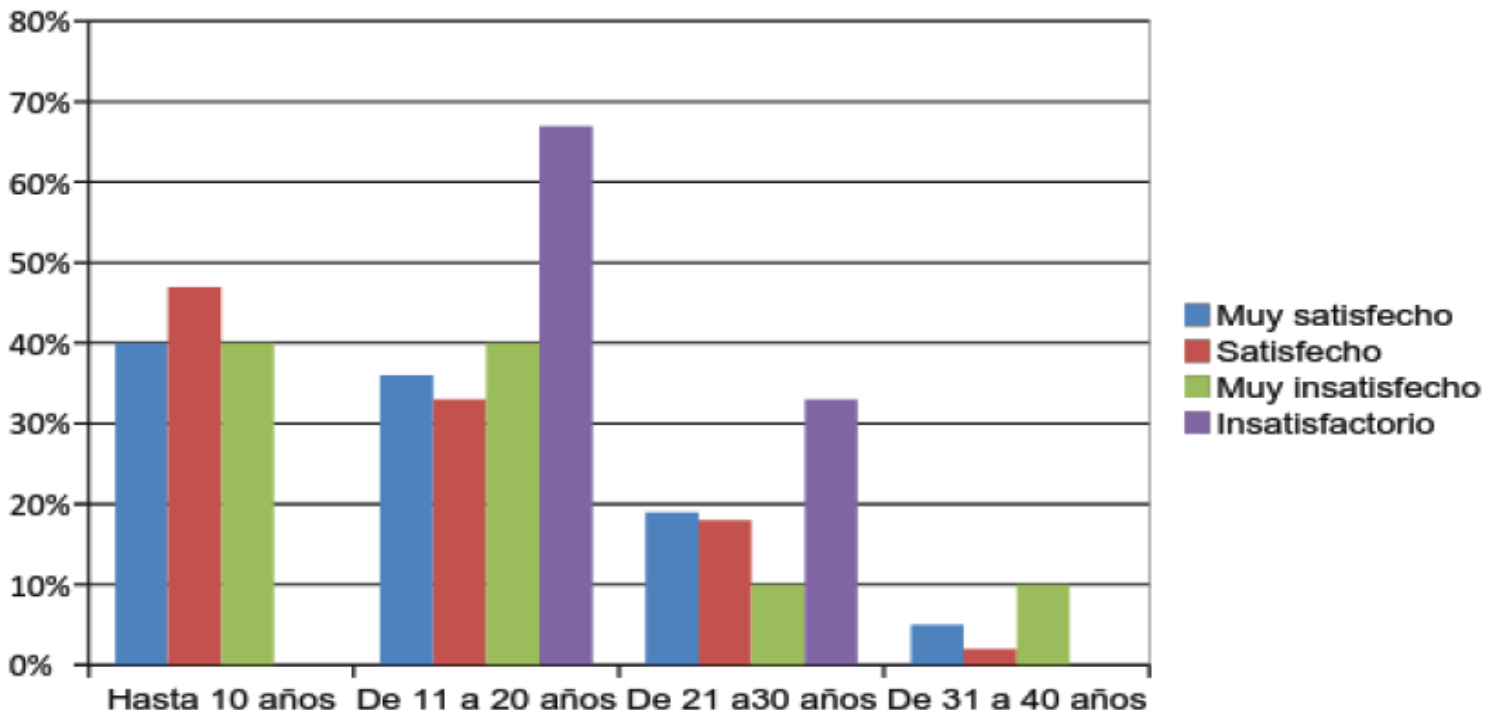

Figura 3. La práctica relacionada a la identidad de ser docente.

La relación entre la práctica docente y la identi- pues son elementos vocacionales y subjetivos dad propia del mismo es estrecha y significativa, los que llevan al desarrollo de dicha identidad.

Tabla 8. El desempeño docente relacionada al tiempo de servicio.

\begin{tabular}{|c|c|c|c|c|c|}
\hline \multirow{2}{*}{ Años de servicio } & \multicolumn{5}{|c|}{ La calidad del desempeño docente está relacionada al tiempo } \\
& \multicolumn{5}{|c|}{$\begin{array}{c}\text { Nada de } \\
\text { en servicio docente }\end{array}$} \\
\cline { 2 - 6 } & $\begin{array}{c}\text { Algo en } \\
\text { desacuerdo }\end{array}$ & $\begin{array}{c}\text { Ni de acuerdo ni en } \\
\text { desacuerdo }\end{array}$ & $\begin{array}{c}\text { Algo de } \\
\text { acuerdo }\end{array}$ & De acuerdo \\
\hline Hasta 10 años & $32 \%$ & $45 \%$ & $54 \%$ & $38 \%$ & $42 \%$ \\
\hline De 11 a 20 años & $55 \%$ & $45 \%$ & $15 \%$ & $35 \%$ & $35 \%$ \\
\hline De 21 a 30 años & $14 \%$ & $9 \%$ & $23 \%$ & $23 \%$ & $15 \%$ \\
\hline De 31 a 40 años & $0 \%$ & $0 \%$ & $8 \%$ & $4 \%$ & $8 \%$ \\
\hline
\end{tabular}

La mayoría de países Centro americanos viven una profundización impresionante de la conflictividad en los sistemas educativos; incluso en aquellos que tradicionalmente han manejado las diferencias entre gobiernos y docentes a través de negociaciones dialogadas. Honduras no es la excepción, y por la importancia que trasciende la satisfacción de ser docente; con base a normas claras y trasparentes, es de suma importancia ponerlo en las mesas de discusión. Es decir, estos conflictos tienen incidencia en causar fragilidad en los sistemas educativos y en su incapacidad para dar respuestas a los problemas estructurales de la Educación. 
Tabla 9. Satisfacción con el proceso de selección

\begin{tabular}{|c|c|c|c|c|c|}
\hline \multirow{2}{*}{ Años de servicio } & \multicolumn{4}{|c|}{ ¿Está satisfecho con el proceso de selección a la incersión laboral? } \\
\cline { 2 - 6 } & $\begin{array}{c}\text { Nada de } \\
\text { acuerdo }\end{array}$ & $\begin{array}{c}\text { Algo en } \\
\text { desacuerdo }\end{array}$ & $\begin{array}{c}\text { Ni de acuerdo ni en } \\
\text { desacuerdo }\end{array}$ & $\begin{array}{c}\text { Algo de } \\
\text { acuerdo }\end{array}$ & De acuerdo \\
\hline Hasta 10 años & $35 \%$ & $54 \%$ & $56 \%$ & $28 \%$ & $42 \%$ \\
\hline De 11 a 20 años & $44 \%$ & $33 \%$ & $44 \%$ & $39 \%$ & $25 \%$ \\
\hline De 21 a 30 años & $21 \%$ & $8 \%$ & $0 \%$ & $28 \%$ & $25 \%$ \\
\hline De 31 a 40 años & $0 \%$ & $4 \%$ & $0 \%$ & $6 \%$ & $8 \%$ \\
\hline
\end{tabular}

Es evidente que el factor antigüedad no inter- de satisfacción entre los docentes cuyo serviviene en la satisfacción del proceso de selec- cio oscila entre los 10 años.

ción; no obstante posee un moderado nivel

Tabla 10. Jornadas de inducción

\begin{tabular}{|c|c|c|}
\hline \multirow{2}{*}{ Años de servicio } & ¿Se le brindó jornada de inducción para la docencia? \\
\cline { 2 - 3 } & Sí & No \\
\hline Hasta 10 años & $33 \%$ & $48 \%$ \\
\hline De 11 a 20 años & $40 \%$ & $35 \%$ \\
\hline De 21 a 30 años & $20 \%$ & $17 \%$ \\
\hline De 31 a 40 años & $8 \%$ & $0 \%$ \\
\hline
\end{tabular}

Gracias al nacimiento de nuevas necesidades centes con menos tiempo de servicio quienes y de la evolución pedagógica, han surgido manifiestan haber recibido guía para el ejerformas mucho más modernas de inducción. cicio de su profesión.

Esto es observable, ya que son aquellos do-

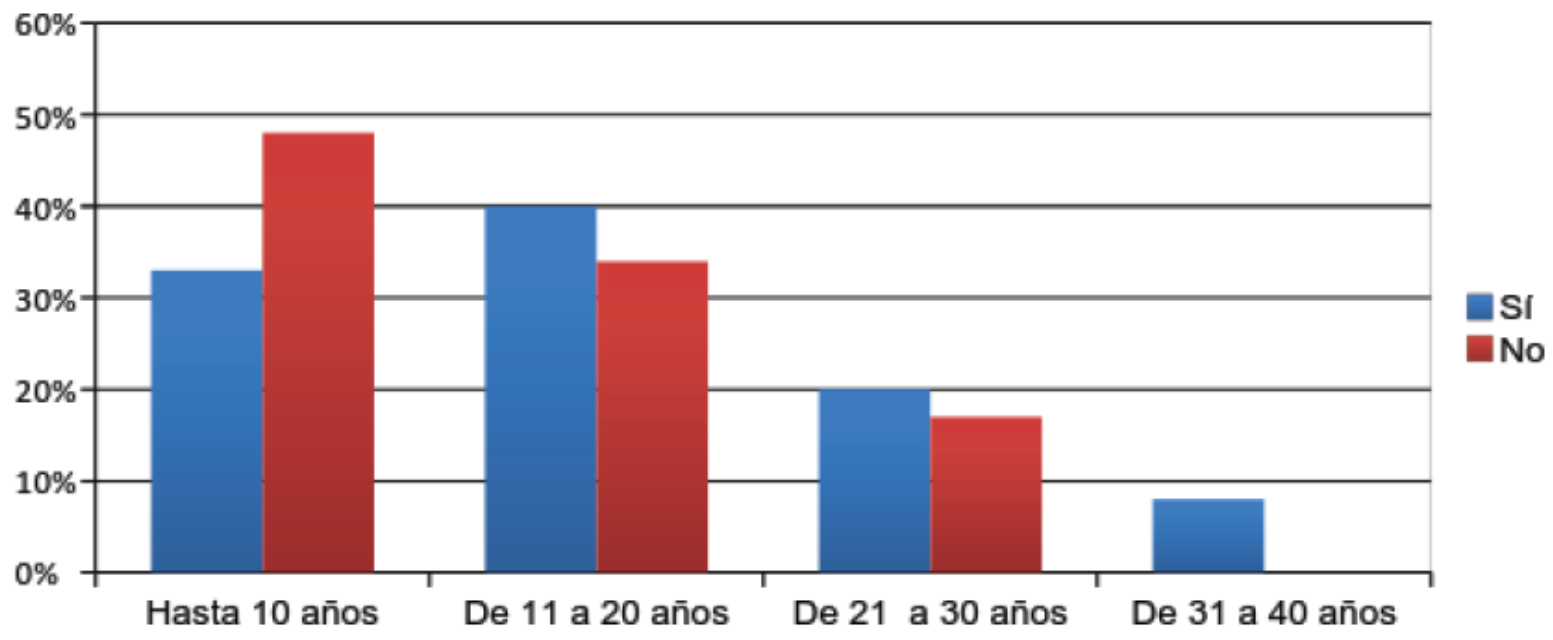

Figura 4. Inducción realizada por la Secretaría de Educación. 
Un $49 \%$ de los docentes con diez años de servicio aseguró no haber recibido inducción por parte de la Secretaría de Educación; frente a un 32\% que manifestó que sí.
Esta instancia sí se preocupó en un inicio por la realización de este proceso, pero luego ya no se aportó un apoyo constante.

Tabla 11. Programas de inserción laboral

\begin{tabular}{|c|c|c|}
\hline \multirow{2}{*}{ Años de servicio } & \multicolumn{2}{|c|}{ Considera importante un programa de inserción laboral } \\
\cline { 2 - 3 } & Sí & No \\
\hline Hasta 10 años & $44 \%$ & $13 \%$ \\
\hline De 11 a 20 años & $33 \%$ & $75 \%$ \\
\hline De 21 a 30 años & $19 \%$ & $0 \%$ \\
\hline De 31 a 40 años & $3 \%$ & $13 \%$ \\
\hline
\end{tabular}

El fortalecimiento de la profesión docente debe ser favorecido con programas de innovación en el desarrollo de nuevas estrategias, enfoques para un mejoramiento integral, cumpliendo metas según programas diseñados a docentes nóveles, intermedios experimentados; todo esto en miras de fortalecer la capacitación continua y el desarrollo de la carrera docente. Existen lecciones aprendidas de docentes experimentados que podrían, en Honduras, ser tutores de docentes nóveles; y se rescataría toda esa riqueza de experiencias enriquecedoras y por supuesto mantener un profesorado en la dinámica del conocimiento.

\section{7 Capacitaciones recibidas en los últimos años de su labor}

Tabla 12. Capacitación sobre programas de formación docente

\begin{tabular}{|c|c|c|}
\hline \multirow{2}{*}{ Años de servicio } & \multicolumn{2}{|c|}{ Conoce cómo está diseñado el programa de formación docente } \\
\cline { 2 - 3 } & Sí & No \\
\hline Hasta 10 años & $40 \%$ & $43 \%$ \\
\hline De 11 a 20 años & $33 \%$ & $40 \%$ \\
\hline De 21 a 30 años & $19 \%$ & $17 \%$ \\
\hline De 31 a 40 años & $7 \%$ & $0 \%$ \\
\hline
\end{tabular}


Tabla 13. Capacitación sobre contextos sociales

\begin{tabular}{|c|c|c|}
\hline \multirow{2}{*}{ Años de servicio } & Conocimiento sobre contextos socioeducativos de los alumnos \\
\cline { 2 - 3 } & Sí & No \\
\hline Hasta 10 años & $50 \%$ & $39 \%$ \\
\hline De 11 a 20 años & $28 \%$ & $42 \%$ \\
\hline De 21 a 30 años & $14 \%$ & $19 \%$ \\
\hline De 31 a 40 años & $8 \%$ & $0 \%$ \\
\hline
\end{tabular}

En la tabla 12 se observa que el conocimiento sobre programas de formación es relativamente equilibrado dependiendo o no de los años de servicio. Mientras que en la tabla 13 Los datos guardan proporción entre el conocimiento y el desconocimiento de contextos socioeducativos de los alumnos, pero divididos en dos grupos: desde la inicial hasta los veinte años de servicio; y de 21 a 40 años de servicio.

Tablas 14. Capacitación sobre currículo escolar

\begin{tabular}{|c|c|c|}
\hline \multirow{2}{*}{ Años de servicio } & \multicolumn{2}{|c|}{ Currículo escolar } \\
\cline { 2 - 3 } & Sí & No \\
\hline Hasta 10 años & $48 \%$ & $38 \%$ \\
\hline De 11 a 20 años & $29 \%$ & $46 \%$ \\
\hline De 21 a 30 años & $17 \%$ & $17 \%$ \\
\hline De 31 a 40 años & $6 \%$ & $0 \%$ \\
\hline
\end{tabular}

Es visible la falta de capacitación sobre el currículo escolar, por lo menos en la mitad de docentes encuestados y con un periodo de menos años de servicio.

Capacitación sobre adecuación curricular para la enseñanza y capacitación sobre cómo crear comunidades de aprendizaje

En términos de capacitaciones son los docentes con menos tiempo de labor quienes han recibido dichas prácticas; pero a la vez, son ellos quienes tienen necesidad de ellas. Esto es visible en los porcentajes proporcionales, específicamente entre aquellos que sí han participado de capacitaciones y los que no las han recibido. Cabe mencionar que la mayor incidencia se ha dado por parte de los docentes que tienen menos de 20 años de servicio, con un porcentaje de $48 \%$ de capacitaciones participadas.

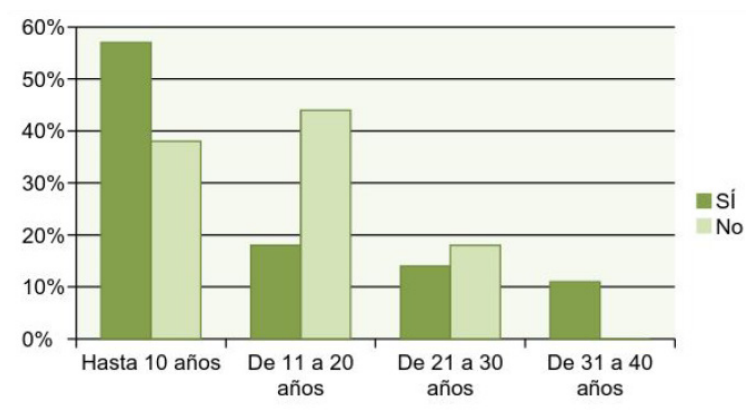

Figura 5. Formación para la supervivencia de ser docente. 
La formación ha sido constante y mejorado en los últimos años, ya que solo un $10 \%$ de los docentes con más tiempo de servicio, un 50\% de los docentes con menos años de servicio sí recibieron la formación.

Tabla 17. Capacitación sobre la identidad de ser profesional de la enseñanza

\begin{tabular}{|c|c|c|}
\hline \multirow{2}{*}{ Años de servicio } & \multicolumn{2}{|c|}{ Identidad profesional } \\
\cline { 2 - 3 } & Sí & No \\
\hline Hasta 10 años & $54 \%$ & $33 \%$ \\
\hline De 11 a 20 años & $26 \%$ & $45 \%$ \\
\hline De 21 a 30 años & $13 \%$ & $20 \%$ \\
\hline De 31 a 40 años & $8 \%$ & $2 \%$ \\
\hline
\end{tabular}

Existe un balance para aquellos docentes menores a 20 años de servicio; no obstante, la importancia medular que posee este tipo de capacitaciones para la vida del profesional de la enseñanza no debe dejarse de lado.

La falta de iniciativa en cuanto a la capacitación profesional es visible en aquellos docentes con más tiempo de servicio. Estas cifras reflejan que aquellos con mayor experiencia en la docente creen que necesitar menos formación. (Ver Figura 6)

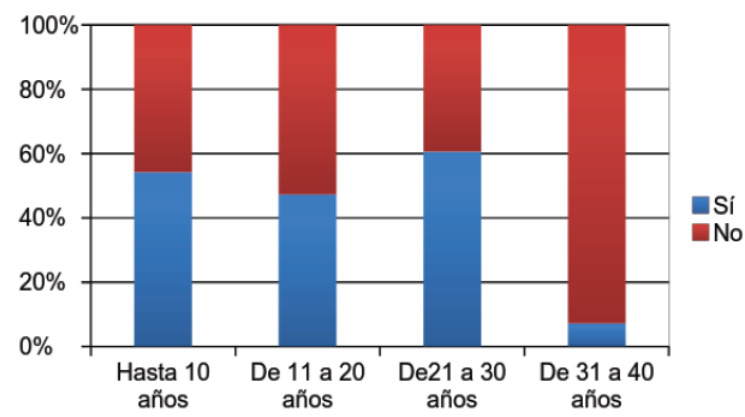

Figura 6. Iniciativas para capacitarse.
Es claro el alto porcentaje de desacuerdo sobre la resolución de las dificultades en la labor docente, ya que en las diferentes áreas de acción predominan los niveles de desacuerdo, especialmente en las áreas urbanas. En cuanto a la importancia de un programa de inserción laboral en las distintas zonas y la formación recibida para la enseñanza, los resultados son bajos en cuanto al nivel de satisfacción. El porcentaje para ambas interrogantes fue de 80\% de insatisfacción. (Ver Figura 7.)

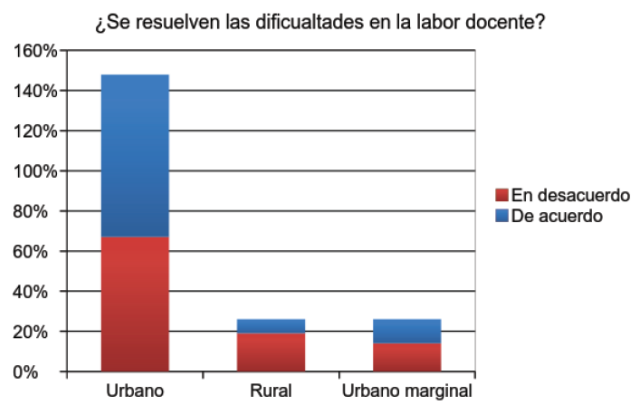

Figura 7. Resolución de problemas de la labor docente por zona. 


\section{Discusión}

Como modelo, la idea de una «sistema de formación con base a una teoría del desarrollo», muchas veces es amenaza a deslizarse hacia la sustitución del fenómeno que busca designar una sociedad imaginada, regida por la lógica de la producción y acumulación del conocimiento. Ello pasa a sustituir en los análisis, a sociedades con problemas y realidades concretos muy diversos; y en la mayoría de los casos, a sociedades privadas de los beneficios a los que la supuesta sociedad del conocimiento debería franquear el acceso. Con todo, no es posible soslayar el atractivo y la importancia de un modelo de desarrollo que sirva como orientador para el diseño y la aplicación de políticas públicas, pero para cuyo logro hay que trabajar todavía, mucho.

Una de las características de la sociedad en la que vivimos tiene que ver con el hecho de que el conocimiento es uno de los principales valores de sus ciudadanos. Este valor de las sociedades actuales está directamente relacionado con el nivel de formación de sus ciudadanos, y de la capacidad de innovación y emprendimiento que éstos posean. Pero los conocimientos, en estos días, tienen fecha de caducidad, obligando a la persona, hoy más que nunca, a establecer garantías formales e informales para que los ciudadanos y profesionales actualicen constantemente su competencia. Se ha entrado en una sociedad que exige de los profesionales una permanente actividad de formación y aprendizaje.

En este sentido el sistema de formación docente es una política válida para hablar de desarrollo humano en un Estado. ¿Cómo y por qué? Quizá sea muy comprometido afirmarlo, pero la experiencia en temas de formación docente, orienta a afirmar que si tuviese un sistema que dirija al docente a educar para el desarrollo humano mediante capacidades; incentivar competencias en cultura escolarizadas y no escolarizadas, se tendría una educación para un bienestar personal y colectivo. Aunado a esto, el definir perfiles de docentes nóveles, intermedios y experimentados que se inserten a la docencia con las competencias definidas en inserción laboral.

Esto no es una simple idea, es pensar en forma holística y darse cuenta cómo están laborando los docentes; cómo se insertaron en el sistema educativo; cómo es la formación docente, es decir, si se les capacita por estratos, nóveles (0 a 5 años), intermedios (de 5 a 15 años) y/o experimentados (de 15 a mas). Son preguntas que orientan a referentes teóricos y prácticos para una nueva investigación. 


\section{Referencias}

Alas S., M. y Moncada G., G. (s.f.). Calidad y equidad en la educación nacional; Estado de la educación nacional, U.P.N.F.M.

Amador, O. y Membreño, J. (1994). Estudio diagnóstico de la organización y funcionamiento de los centros de aprendizaje docente en III encuentro de investigadores en educación. Tegucigalpa.

Barahona, L.; Casaca, Z.; Mejía, R. e Isaula, S. (1997). Investigación evaluativa sobre docente el funcionamiento de los centros de aprendizaje Secretaría de Educación, Comayagüela.

Carden, F. (2009). Del conocimiento a la política; Del aprovechamiento de la investigación para el Desarrollo; Junio 2009; España.

Chávez, M. y otros (1997). La formación inicial de docentes de educación básica en Honduras. Tegucigalpa.

De Souza Silva, J. (2005). La innovación de la innovación institucional: De lo universal, mecánico y neutral a lo contextual, interactivo y ético desde una perspectiva latinoamericana, 2005.

Díaz, Q. (2010). La unidocencia: desafíos y retos con la aplicación del diseño curricular nacional básico (DCNB) en la formación permanente en el marco del sistema nacional de formación docente (SINAFOD). Revista Centro Americana de Educación; Patria Grande, CECC- SICA.

Documento extraído del Sistema Nacional de Formación Docente. (2009 )INICE, Pág.4, Honduras. Guadamuz, L; Ramírez, M.; Posas, M. y Boquín, L. (1999). Honduras: Evaluación del plan nacional de acción para todos. SE/BM/FNUAP/PNUD/UNESCO/UNICEF.

Hargreaves, A. (2003). Profesorado, cultura y postmodernidad, Cambian los tiempos, cambia el profesorado; Ediciones Morata, SL.

Informe de Desarrollo Humano (2013) PNUD.

Imbernón, F. (2004): La formación del profesorado: ciertas confusiones y algunas evidencias. Aula de Innovación Educativa, Revista española.

Imbermón, F. (2007). La formación permanente del profesorado: Nuevas ideas para formar en la innovación y el cambio, Colección ideas clave: Editorial GRAÓ, de IRIF, España.

Marcelo, C. (2011). Los comienzos en la docencia: un profesorado con buenos principios, Revista de currículum y formación del profesorado, Universidad Sevilla, España. 
Medina, J. L. (2008). Material fotocopiado sobre "Técnicas de Grupo Colaborativo"; Universidad de Barcelona España.

Moncada, G., Hernández, R. y Perdomo, I. (2007) Diagnostico de desarrollo de capacidades para la implementación EFA. GTZ/UPEG/SE. Tegucigalpa.

Pavón, R. (2003). El rendimiento académico de los docentes de alumnos docentes participantes en el Programa de Formación Continua: 1998-2000. Revista Paradigma. 12, 34-45.

PREAL (2005). Informe del Progreso Educativo de Honduras: El futuro es hoy. Tegucigalpa. FEREMA.

Revista de Investigación Educativa "Perspectiva Global”; Depto. de Investigación, INICE; Año 2, No.- 2, Diciembre, 2008.

Revista Pensamiento Educativo (2007). Mejorando la formación y el desarrollo profesional docente en Latinoamérica Vol. 41, № 2.

Rodríguez, G. y García J. (1999). “Metodología de la investigación cualitativa”, Editorial Aljibe, Málaga, España.

Sánchez, S. (1998). "Fundamentos para la investigación educativa”, Editorial Magisterio, Santa Fe de Bogotá.

SE/GTZ (1997). Educación y Desarrollo. Estudio Sectorial. Plan Decenal. Tegucigalpa.

Stenhouse, L. (1987). “QQué es un currículum?, El currículum hipotético”. pp. 103- 107. En Stenhouse, L. "La investigación como base de la enseñanza", Ediciones Morata, Madrid.

Torres, R.M. (2000). De agentes de la reforma a sujetos del cambio: la encrucijada docente en América Latina. Perspectivas, XXX.

Unidad Externa de Medición de la Calidad de la Educación (UMCE) (1998). Factores asociados al rendimiento académico (español y matemáticas de tercero y sexto grado). Tegucigalpa, UMCE.

Weiis, C. H. (2009). Del conocimiento a la política .Máximo aprovechamiento de la investigación para el Desarrollo. Editorial Barcelona. 